\title{
Regulation of cell-cell contacts in developing Drosophila eyes by Dsrc41, a new, close relative of vertebrate c-src
}

\author{
Fumitaka Takahashi, Sachiko Endo, Tetsuya Kojima, and Kaoru Saigo ${ }^{1}$ \\ Department of Biophysics and Biochemistry, Graduate School of Science, University of Tokyo, Bunkyo-ku, Tokyo 113, \\ Japan
}

In Drosophila, Dsrc64 is considered a unique ortholog of the vertebrate c-src; however, we show evidence to the contrary. The closest relative of vertebrate c-src so far found in Drosophila is not Dsrc64, but Dsrc41, a gene identified for the first time here. In contrast to Dsrc64, overexpression of wild-type Dsrc41 caused little or no appreciable phenotypic change in Drosophila. Both gain-of-function and dominant-negative mutations of Dsrc41 caused the formation of supernumerary R7-type neurons, suppressible by one-dose reduction of boss, sev, Ras1, or other genes involved in the Sev pathway. Dominant-negative mutant phenotypes were suppressed and enhanced, respectively, by increasing and decreasing the copy number of wild-type Dsrc41. Colocalization of Dsrc41 protein, actin fibers and DE-cadherin, and Dstc41-dependent disorganization of actin fibers and putative adherens junctions in precluster cells suggested that Dsrc41 may be involved in the regulation of cytoskeleton otganization and cell-cell contacts in developing ommatidia.

[Key Words: Protein tyrosine kinase; Drosophila; src; Dsrca1; cadherin; boss]

Received January 30, 1996; revised form accepted May 28, 1996.

The Src tyrosine kinase family in vertebrates consists of nine members (for review, see Cooper and Howell 199.3), at least three of which-src, yes, and fyn-are widely expressed in a variety of cells including those in the neural ectoderm and developing cerebellum /Cotton and Brugge 1983; Fults et al. 1985; Manness et al. 1986; Zhao et di. 1990). These tyrosine kinases may participate in developmental processes such as ncural and hematopoietic cell differentiation (Molina et al. 1992; Yagi et al. 1993). Studies using knockout mice suggest that compensatory interactions occur between Src family tyrosine kinases. Only a restricted phcnotype, or no overt dysfunctional consequences is observed in a loss-of-function mutant of src, yes, and fyn, whereas most $s r c / f y n$ or src/yes double mutants die perinatally and a considerable number of fyn/yes double mutants undergo degencrative renal damage with consequent diffuse segmental glomeruloscleosis (Stein et al. 1994).

Elimination of the conserved tyrosine residue in the carboxyl terminus endows Src family tyrosine kinases with transforming activity. This activity is thought to be induced by derepression of tyrosine kinase activity (Cartwright et al. 1987), which is normally repressed via stoichiometric phosphorylation of the carboxy-terminal ty-

${ }^{1}$ Corresponding author. rosine residue by tyrosine kinase kinases such as Csk. Individuals with homozygous deficiency of csk are embryonic lethal (Nada et al. 1993; Imamoto and Soriano 19931. Because of this repression mechanism, the overexpression of $\mathrm{c}$-src in fibroblasts has no significant effect on cell growth or morphology, nor overall abundance of tyrosine-phosphorylated proteins iShalloway et al. 1984; Iba et al. 1985).

In Drosophila, three src-related genes, Dash (Henkemeyer et al. 1988), Dsrc29 (Gregory et al. 1987), and Dsrc64 (Simon et al. 1985), have been identified using a $\mathrm{v}$-src fragment as a probe. Only the polypeptide encoded by $D s r c 64$ (Dsrc64) resembles, in its entire structure, vertebrate Src and is presumed to belong to the Src family (Simon et al. 1985). Dash may be a counterpart of vertebrate abl (Henkemeyer et al. 1988), whereas Dsrc29 is suggested to be in the same family as mammalian tyrosine kinase genes itk and tec (Tsukada et al. 1993). Recently, however, Kussick et al. (1993) showed Dsrc64 to have properties considerably different from those of vertebrate c-Src. In contrast to vertebrate c-Src overexpressed in fibroblasts (Iba et al. 1985), wild-type Dsrc64 overexpressed in Drosophila was phosphorylated not only at the carboxy-terminal tyrosine residue but at major in vitro autophosphorylation sites as well (Kussick and Cooper 19921, causing embryonic lethality or abnormal differentiation (Kussick et al. 1993). Thus possibly, 
carboxy-terminal tyrosine phosphorylation of Dsrc64 is substoichiometric (Kussick and Cooper 1992).

Here, we show isolation and characterization of Dsrc41, a new src-related gene in Drosophila. Nucleotide sequence analysis and analysis of phenotypes of gain-of-function and dominant-negative mutations in developing retina and embryos suggested that Dsrc41 is the closest relative of vertebrate c-src found so far in Drosophila and may play an essential role in cytoskeleton organization and cell-cell contacts in development.

\section{Results}

Molecular cloning of Dsrc41, the closest relative of vertebrate c-src found so far in Drosophila

$d t k-5$ is a PCR fragment encoding a partial tyrosine kinase sequence (Shishido et al. 1991). During the course of searching $d t k 5$-related genes in Drosophila, we discovered two $\lambda$ clones, $\lambda g f k 1$ and $\lambda g f k 2$ (Fig. 1A), which together encode the putative polypeptide showing much greater similarity in amino acid sequence to vertebrate c-Src than Dsrc64 (see Fig. 2B). As its cDNA hybridized at $41 \mathrm{~B}$ or $41 \mathrm{C}$ on the second chromosome /data not shown), we refer to the gene hereafter as Dsrc41.

Nucleotide sequences of the longest cDNA and relevant genomic DNA fragments were determined (Fig. 2A). The longest cDNA was $2.9 \mathrm{~kb}$ in length, which would agree well with the 3-kb RNA observed in Northern blots (Fig. 1B). Dsrc41 contains 9 exons that would code for a polypeptide of 517 amino acid residues (Figs. 1A and 2A). The Dsrc41 gene product (Dsrc41) resembled the vertebrate Src family kinase consensus over the entire SH1 (catalytic), SH2, and SH3 domains, the amino-terminal myristylation site, and the carboxy-terminal tail (Fig. 2A,B).

As shown in Figure 2B, the kinase domain of Dsrc4l was much more similar in amino acid sequence to those of vertebrate Src members than Dsrc64. On average 173 amino acids were conserved between Dsrc4l and five vertebrate Src members (Src, Yes, Yrk, Fgr, and Fyn), whereas only 148 amino acids were invariant between Dsrc4l and Dsrc64. Similarity in amino acid sequence between Dsrc4l and vertebrate Src members was also evident in SH3 and the carboxy-terminal tail (Fig. 2B), making possible unequivocal assignment of Tyr-511 of Dsrc41 to c-Src Tyr-527, whose phosphorylation is essential for the regulation of kinase activity within cells (Cartwright et al. 1987). Structural resemblance between

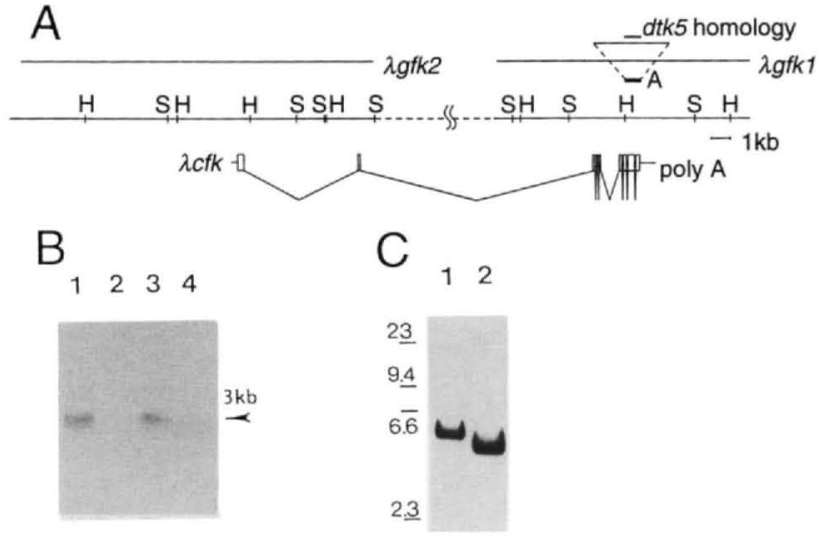

Figure 1. (A) Genomic and cDNA structures of Dsrc41. \{ $\lambda g f k 1$ and $\lambda g f k 2)$ Genomic clones; $(\lambda c f k)$ the longest cDNA clone. (Open boxes) Presumed coding regions. (Bar A) Hybridization probe. (H) HindIII; (S) SalI. (B) Northern blotting of Dsrc41 RNA. (Lanes 1-4) RNA extracted from embryos, total larvae, total pupae, and adults (males and females), respectively. (Arrowhead) 3-kb Dsrc41 transcript. (C) Southern analysis of Dsrc41. Canton-S adult DNA was terminally digested with EcoRI (lane 1) and HindIII (lane 2). Probe, fragment A in A.

vertebrate c-src and Dsrc41 can be extended further to exon-exon junctions (Fig. 2E). In contrast, no correlation in exon-exon junctions was detected between Dsrc64 and c-src. Dsrc64 thus likely forms its own group distantly related to Dsrc41 and vertebrate src family members (Fig. 2C).

Drosophila may contain no close relative of Dsrc41, because as shown in Figure 1C, no band other than those for Dsrc41 could be detected by hybridization under less stringent conditions.

\section{Temporal and spatial expression of Dsrc41 RNA}

Poly $(\mathrm{A})^{+}$RNA extracted from embryos, larvae, pupae, and adults was examined by Northern blotting (Fig. 1B). A single transcript, $3 \mathrm{~kb}$ long, was found in both embryos and pupae. Its spatial patterns in embryos and imaginal discs were also examined, as shown in Figure 3. In very early embryos $\{\mathrm{A}\}$, little or no Dsrc41 RNA was detected, suggesting the level of its maternal expression to be quite low, if any. Dsrc41 RNA was first discernible at stages 2 or 3 (Campos-Ortega and Hartenstein 1985). At

Figure 2. (A) Nucleotide sequence of the Dsrc41 gene and the deduced amino acid sequence of Dsrc41. Lowercase letters show the 5 '-genomic flanking sequence. Overlines $a$ and $b$ indicate putative TATA-like sequence and the RNA start, respectively. ( $)$ Positions of introns. SH3, SH2, and SH1 domains are boxed. The lysine residue for ATP binding and two regulatory tyrosine residues are circled. The GSDB/DDBJ/EMBL/NCBI accession no. for the Dsrc41 sequence is D42125. (B) Sequence homology in SH1. Residues identical to the counterparts of Dsrc41 are shaded. Amino acid sequences of c-Src (chicken), Yes (human), and Dsrc64 are from Hanks et al. (1988). Broken underlines indicate the SHI domain. (C) A phylogenetic tree of the src family. The location of Dsrc41, which was calculated based on amino acid sequence homology, was superimposed on the phylogenetic tree (Hanks et al. 1988). Thus, the length and exact branching position of the broken line are inaccurate. $(D)$ Structures of various P-based $s r c$ constructs. $(E)$ Conserved exon-exon junctions. 
-120 aagaggacgatatttgagttaaaatgtgaatacggatgccagcccaactaataatagtgcaatcgcgagtcacccatttaatctgcctagtttgaattcttttttgcggccccaacgc CGGTACACAGCTGGATTTTTGTTGGTTGCCAGCAGTCCAGCATCACGCACACCCACACGAGCAAATGTAGATAAAAGCGAGAAAACACTCACGCACGCACACACACAGGCACACGTGTGG 121 TCGTGGGCAAGCATTTA.ATTTCGTTAGTGCCCAGTAAAACAGAGTTGAGCGAAAAGGCGATAGCA.ACTTGCACAAATTGTAAGCAGTGCAGAGAGTCAGTGAAGAGGGGATCGAGTGAGA 241 GCGGCGTTTAGTGCCAACACCCAAGCGCTGATCTCGCAGCTACAAACAATAAACAAACAGTGAAAGTAACAGTAAACGCAAGCGCTGCAATTTGGCTTCCATTGATTGCATTTAGTACTC 361 AGTCTCGGCGGCACACAACGTACTCAGCCAACAATGGGTAACTGCCTCACCACACAGAAGGGCGAACCCGACAAGCCCGCAGATCGA.ATCAAGCTGGACGACCCGCCCACCATCGGAGTC

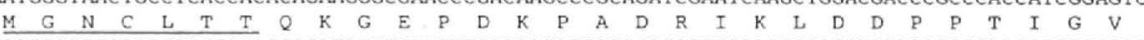

481 GGAGTGGGCGTGCCACAAATCCCCATGCCCTCACACGCCGGACAGCCACCGGAGCAGATACGTCCGGTTCCCCAGATCCCGGAGAGCGAAACGGCAGGTGCCAACGTCAAGATTTTTGTA

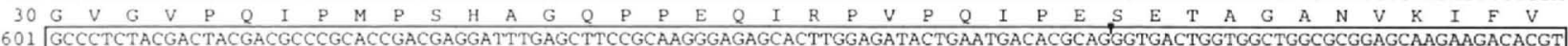

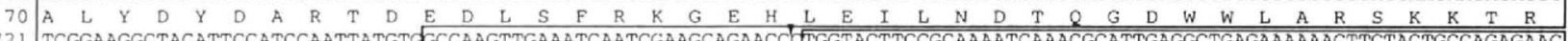
841 GAGCACGGTGCATTTTTAATTCGCGATTCCGAAAGCCGTCACAACGACTACTCGCTATCAGTGCGCGATGGCGATACGGTTAGCATTATCGCATCAGACAATTGGACGAAGGCGGCTTC

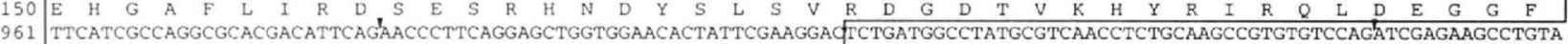

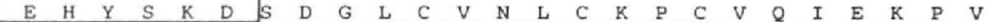
1081 ACTGAGGGGCTTTCGCACCGCACTCGCFATCAGTGGGAGATCGACAGAACATCCTTGAAATTCGTGCGCA.AACTGGGCTCCGGACAGTTTGGCGATGTCTGGGAGGGATTGTGGAACAAC

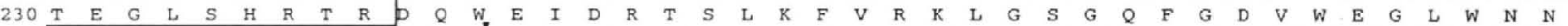
1201 ACAACACCTGTCGCANATMAAACTCCTGAAATCTGCCACAATGGACCCCAAGGATTTCTTAGCGGAAGCCCAGATCATGAAGAAACTGCGCCACACCAAGCTTATACAGTTGTACG

270 T T P A I K T L K S G T M D P K D F L A E A O I M K K L R H T K I I Q 1321 TGCACTGTTGAGGAGCCTATCTATATTATCACAGAGTTAATGAAGCACGGTTCACTGTTGGAATATCTCCAAGCCATTGCAGGCAAGGGTCGTAGCCTTAAAATGCAAACTCTGATTGAT

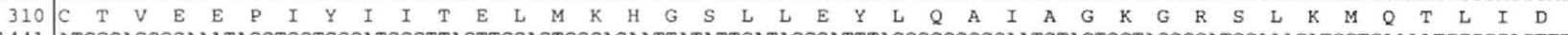
1441 ATGGCAGCGCAAATAGCTGCTGGCATGGCTTACTTGGAGTCCCAGAATTATATTCATAGGGATTTAGCGGCGCGCAATGTACTGGTAGGCGATGGAAACATCGTCAAAATCGCCGACTTT S

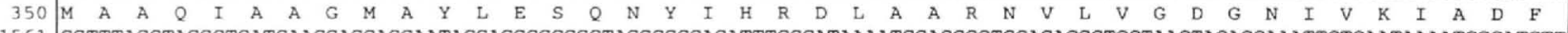
390 G L A R L I K E D E 1681 TGGAGCTTTGGCATTCTTCTCACAGAACTGGTCACCTACGGACGCATACCATATCCAGGCATGACCAACGCTGAGGTGCTAACGCAAGTGGAGCACGGCTATCGAATGCCGCAACCTCCC

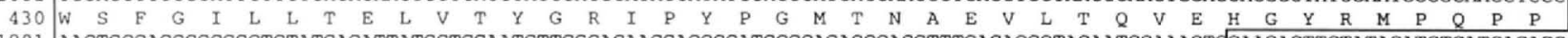
1801 AACTGCGAGCCGCGCCTGTATGAGATTATGCTGGAATGTTGGCACAAGGACCCCATGCGCAGACCCACGTTTGAGACGCTACAATGGAAACT GAAGACTTCTATACATCTGATCAGAGC

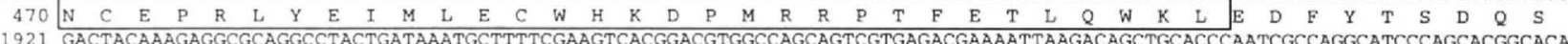
510 D Y K E A Q A Y .

2041 ATTCTTACTAAGTTACTTTTGTTTTGCATACATTTTCTACTGCTGCACAACGTGTCCATGGGCCCCCGTTTGGTGCCCGTTGGCTGTGGTTGTCTAGGTACCGATACGATTCATGTTATT 2161 AGCATTTAAGGTAAATAGTTGCTGAGGTGGAATGCAATTTCAAATGACGTTCCATTGGAACATTCGTTTTCTTGTTTATTATGACAAACATATTGGCACTTGGTCGGATTTATACGCTTT 2281 GACATCCTTGTCGTATATTTCGATTAATATATCAAATATATTATTATTATATATAACCGCATATAGCATATATTTACGCGTATACACACACATATATATATATATGATCACTTATATACA 2401 AATATAAATACAACCGTATAGTCACACCTTCATATGCCGAGACCCTCATTATTGAAGTATGCTAAAACATACTTTACTTCCCTATTTTTCTTTAGTTGATAGGCCAAACTGAGAACGATA 2521 TTTGCAGCACACACATATACAAAAAATTTTCACACTTAATTAATTTCAATTTAATTAATTACGTACGTGTCTTACTTCTATTCTCACTTAACATTATCACCGACATTATATGCTTTATCA 2641 CAAATAGAAAAACCAACACAAACAGCCAACTCATTAAATATATGCTAGCTACTGGATACTTTGCAAGCCCAATAGCTAAATCCATATAATTACTTATTTAGAATTTAAATGTAATTTGTA 2761 ACCTTAACATAATATGCATAAACAAACATACATATACGAAAACATGTACATTTAGAACCAAAAAAATATTTCTTTATAATTTATAAATATAAAATTCTTTAAAAAAAAAAAAAAAAAA

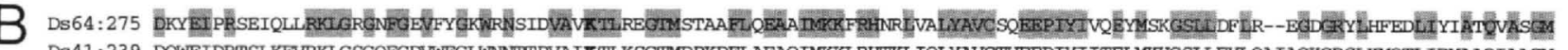
DS41: 239 DQWEIDRTSLKFVRKLGSGQFGDVWEGLWNNTTPVAIKTLKSGTMDPKDFLAEAQTMKKLRHTKLIQLYAVCTVEEPTYIITELLMKHGSLLEYLQAIAGKGRSLKMQTLIDMAAQIAAGM

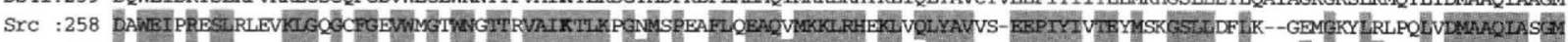

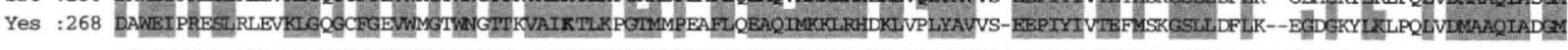

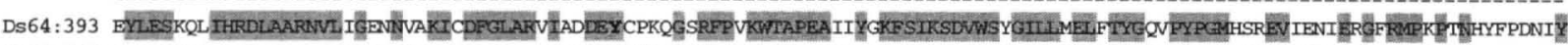
DS41:359 AYLESQNYIHRDLAARNVLVGDGNIVKIADFGLARLIKEDEY EARVGARFPIKWTAPEAANYSKESIKSDVWSFGILLTELVTYGRIPYPGMINAEVLTQVEHGYRMPQPPNC--EPRLY

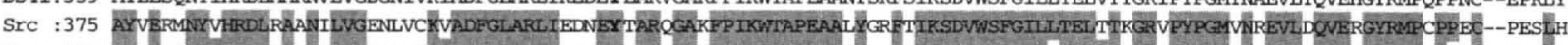

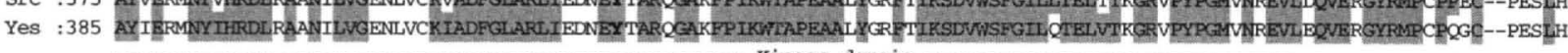

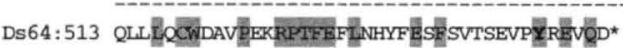

DS41:477 EIMLECWHKDPMRRPTFETL QWKLEDFFYTSDQSDYKEAQAY*

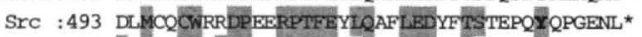

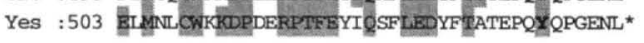

D
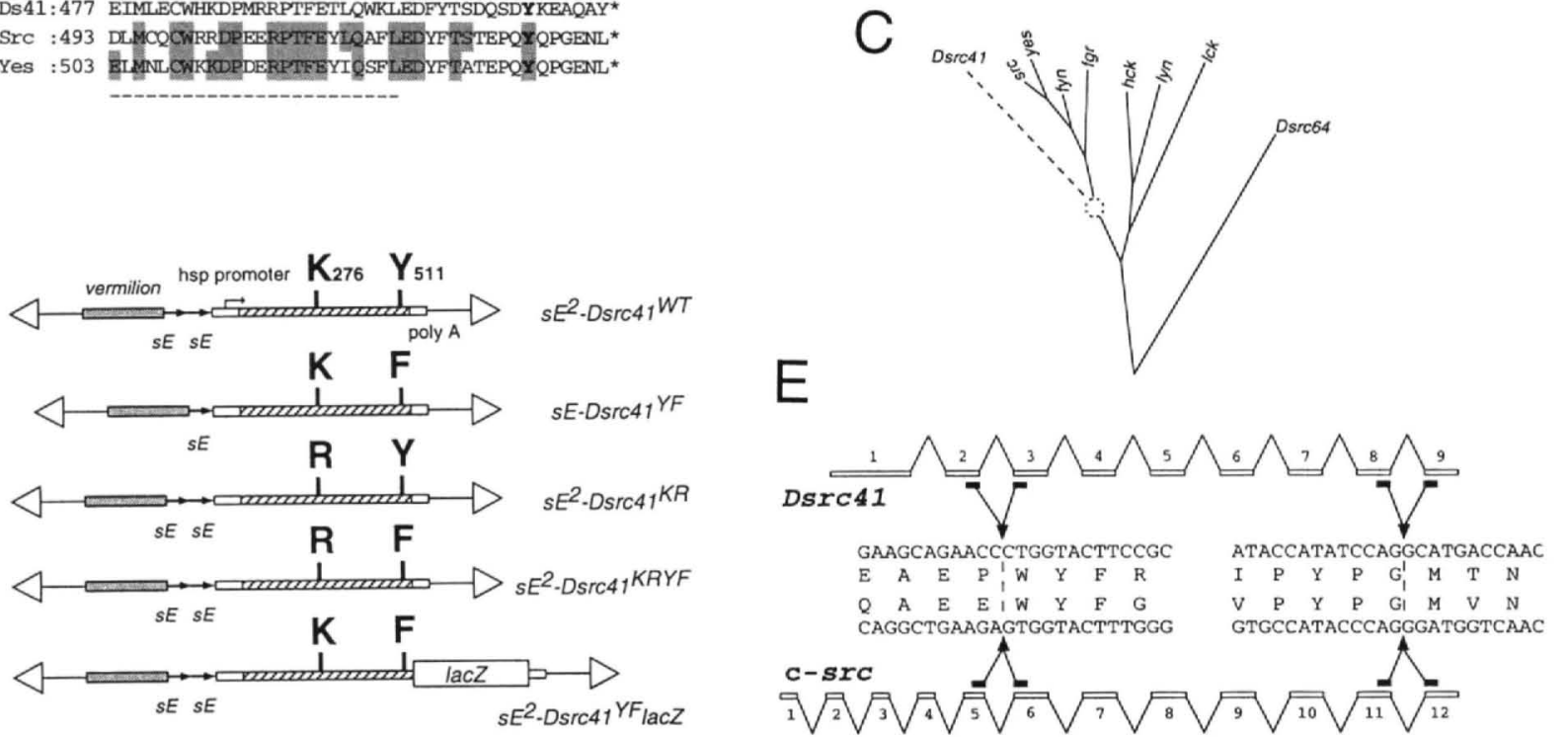

Figure 2. (See facing page for legend.) 

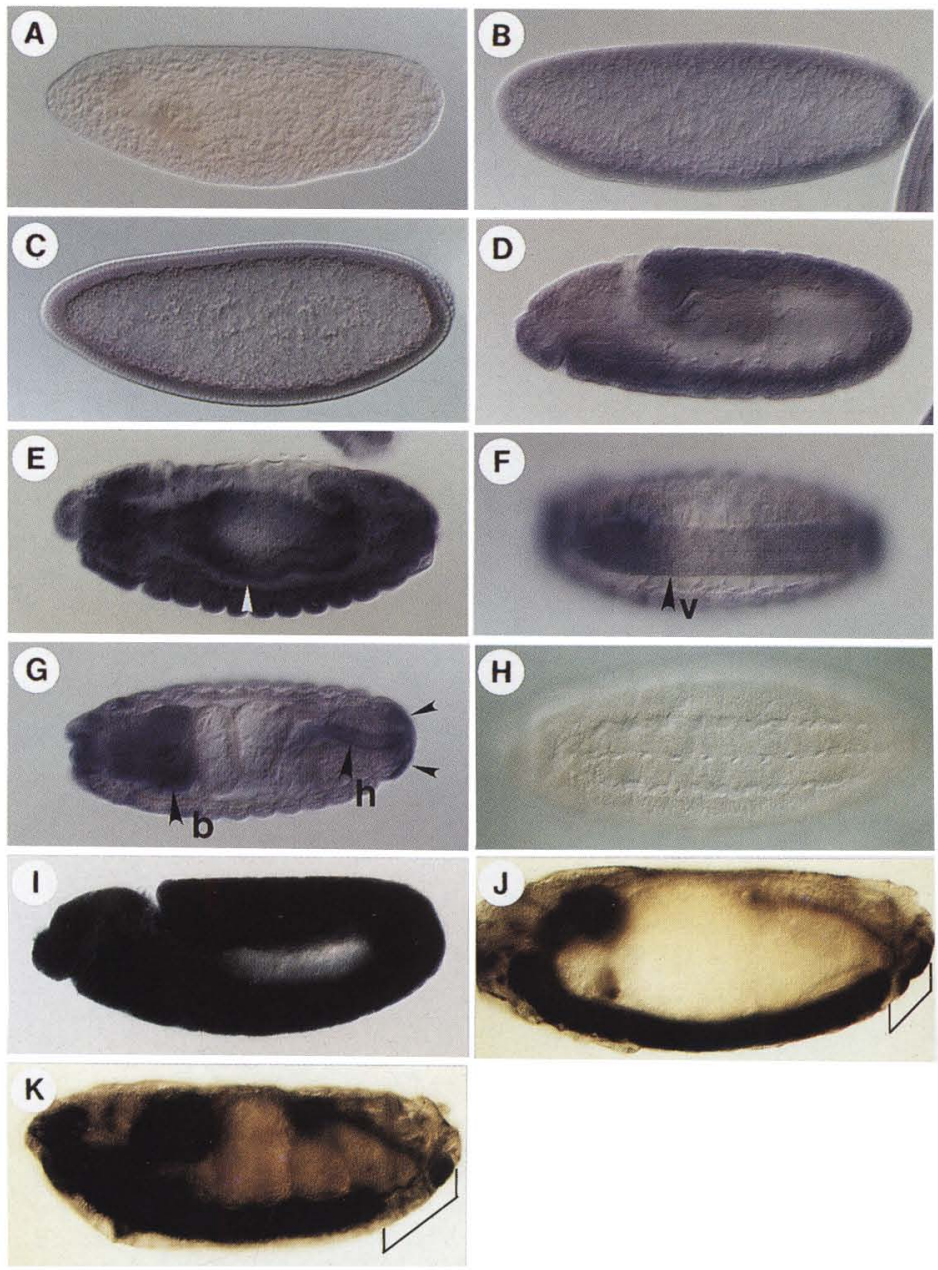
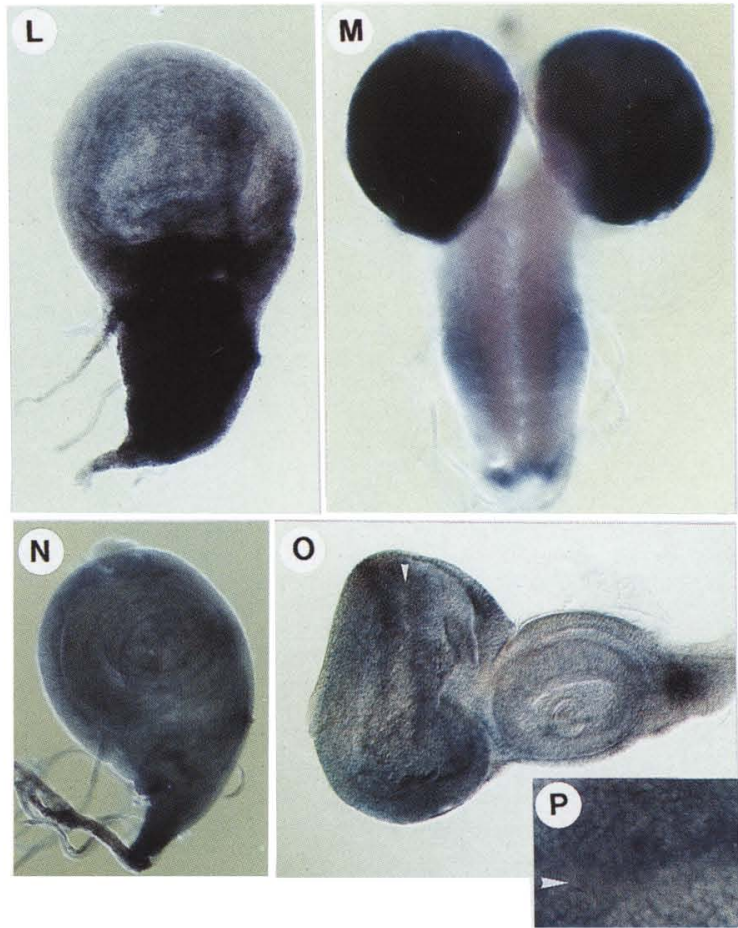

Figure 3. Spatiai and temporal expression of Dsrc4l RNA. In embryos, anterior is to the left. $\{A \mid$ Very early stage. (B) Syncytial blastoderm. (C) Cellular blastoderm. (D) Germ band-extended stage. $(E)$ Stage 13 . The arrowhead indicates the visceral mesoderm. $\mid F, G)$ Stages 15-16. Arrowheads $b, h$, and $v$ indicate brain, hindgut, and ventral ganglions, respectively. The small arrowheads in $G$ indicate

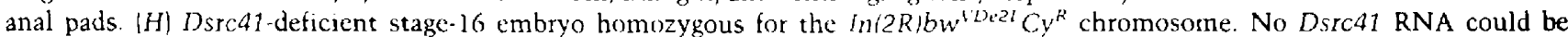
detected. (I) A heat-shocked embryo homozygous for the Dsrc $41^{117}$ insertion (WT-A). $|J, K| S E-D s r c 41^{\text {Yt }}$ and wild-type embryos treated with heat shock at $37^{\circ} \mathrm{C}$ for $30 \mathrm{~min}$ at $4-7 \mathrm{hr}$ of development and then cultured at $25^{\circ} \mathrm{C}$ until $13-16 \mathrm{hr}$ of development. CNS was stained with anti-HRP. Brackets show CNS shortening to be blocked in $1 .\langle L-P|$ Third-instar wing disc $\{L\}, C N S|M|$, leg disc $\{N \mid$, eye-antennal dise $|O|$, and an enlargement of $O\{P \mid$. Antersor is at the top. (Arrowhead) Morphogenetic furrow.

the cellular blastoderm stage, it was expressed weakly in virtually all cells $(\mathrm{C})$. Uniform but somewhat stronger expression persisted until the end of germ-band retraction (D). During stages 13-16, relatively high expression was recognized in visceral mesoderm $|E|$, hindgut, brain, anal pads, and ventral ganglions $(F, G)$. No signal of Dsrc41 RNA was detected in embryos homozygous for a deficiency of Dsrc41 $(\mathrm{H})$. In third-instar larvac, Dsrc41 RNA expression was still strong in the central nervous system (CNS) (M). Almost all cells expressed Dsrc41 RNA in wing $\{L\}, \operatorname{leg}\{N \mid$, and eye-antenna $\{O, P\}$ discs. In contrast, Dsrc64 expression in eye discs has bcen reported to be restricted to photoreccptor precursors posterior to the morphogenetic furrow (Kussick et al. 1993).
In vivo functions of Dsrc41 and vertebrate c.Src are similarly regulated by the carboxy-terminal conserved tyrosine residue

Overexpressed c-src in fibrohlasts induces no appreciable phenotypic change (Shalloway et al. 1984), whereas elimination of the carboxy-terminal tyrosine residue results in activation of intrinsic catalytic activity and malignant transformation (Cartwright et al. 1987). Using Drosophila compound eye and embryonic systems, we examined effects of the overexpression of Dsrc 41 with or without the conserved carboxy-terminal tyrosine residue.

DNA fragments containing either wild-type Dsrc41 
(Dsrc $41^{+}$) or a constitutively active mutant containing a Tyr $\rightarrow$ Phe substitution at position $511\left(D s r c 41^{Y F}\right)$ were subcloned into P-based expression vectors possessing one or two sevenless (sev) enhancers $\left(s E\right.$ or $\left.s E^{2}\right)$ associated with the $h s p 70$ promoter (Fig. $2 \mathrm{D}$ ); $s E^{2}$ is more active than $s E$ (Bowtell et al. 1991). Thus, the expression of the inserted Dsrc 41 gene is bipartite; the hsp 70 promoter enables heat shock-inducible, ubiquitous expression, whereas sev enhancers drive high expression in a limited species of ommatidial cells. Using these constructs, three and two transformant lines containing $s E^{2}$ Dsrc41 ${ }^{+}$(WT-A, WT-B, and WT-C) and $s E-D s r c 41^{Y F}$ (YF-A and YF-B), respectively, were obtained. In situ hybridization showed these transgenes to be overexpressed in the region posterior to the morphogenetic furrow in eye discs and heat-shocked embryos (Fig. 3I).

As with flies with activated Sev tyrosine kinase (Sev ${ }^{S 11}$; Basler et al. 1991), Dsrc41 ${ }^{Y F}$ flies exhibited rough eyes (Fig. 4B) associated with extra R7-type neurons resistant to the (outer rhabdomeres absent) (ora) mutation (Stark and Sapp 1987) (Fig. 5B,E). An ommatidium heterozygous for the $s E-D s r c 41^{Y F}$ transgene insertion (YFA) contained two to three R7-type neurons (Table 1), and the value increased with increasing copy of the inserted transgene (data not shown). In contrast, no appreciable
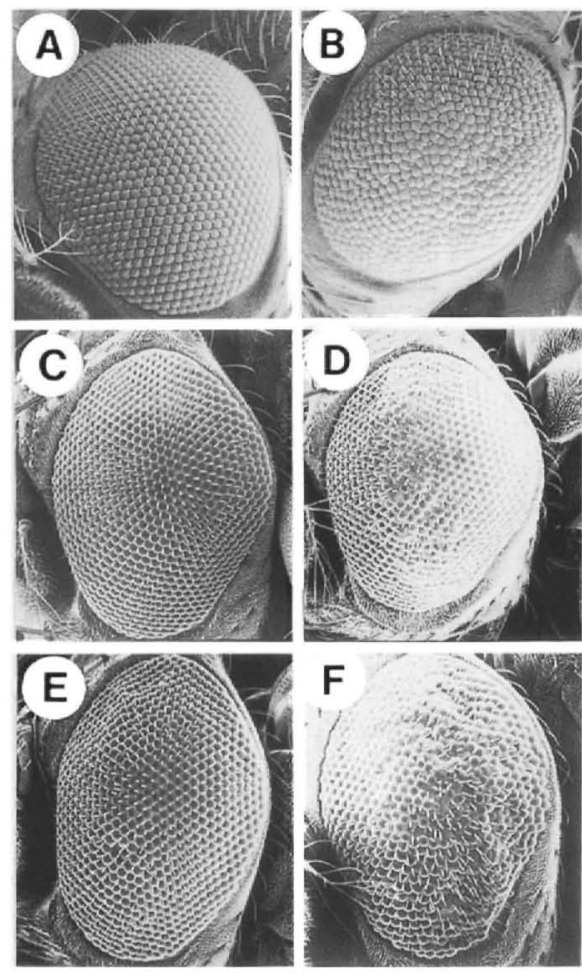

Figure 4. Scanning electron micrographs of compound eyes. $(A)$ Wild type. $(B)$ Heterozygote for $s E-D s r c 41^{\mathrm{YF}}$ insertion (YFA). (C) Homozygote for $s E^{2}-D s r c 41^{+}$insertion (WT-A). (D) Heterozygote for $s E^{2}-D s r c 41^{K R}$ insertion (KR-A). $|E\rangle$ Double het erozygote for $s E^{2}-D s r c 41^{K R}$ and $s E^{2}-D s r c 41^{+}$insertions. $|F|$ Double heterozygote for $s E^{2}-D s r c 41^{K R}$ insertion and a Dsrc4l deficiency.
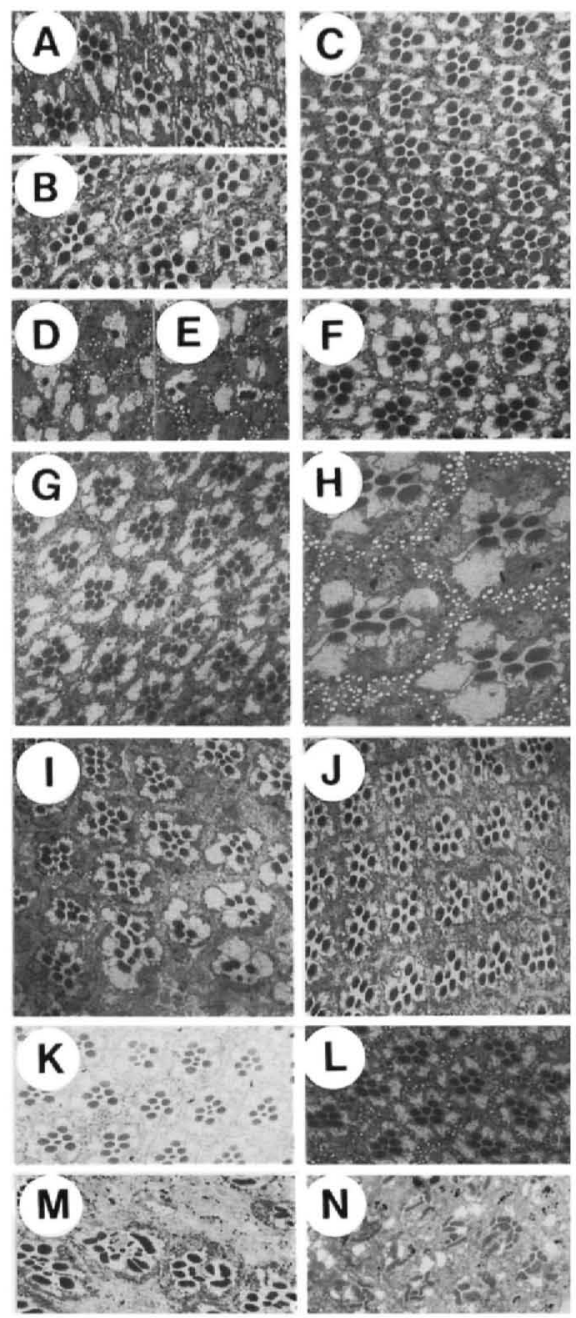

Figure 5. Tangential sections at the $\mathrm{R} 7$ level. $\{A \mid$ Wild type. $(B)$ A transformant line (YF-A) heterozygous for the $s E-D s r c 41^{Y F}$ insertion. (C) A transformant (WT-A) homozygous for the $s E^{2}$ Dsrc $41^{+}$insertion. (D) Outer photoreceptor rhabdomeres are absent from the ora mutant. (E) Supernumeral rhabdomeres in YF-B are stable against the ora background. $(F-H)$ Fly strains doubly heterozygous for $s E-D s r c 41^{Y F}$ insertion (YF-A) and one of a mutation of sevenless pathway components. $|F| \operatorname{Ras} 1^{e 2 F} .(G)$ boss ${ }^{\prime}$. (H) $\mathrm{sev}^{d 2}$. (I) Heterozygote for $s E^{2}-D s r c 41^{K R}$ insertion (KR-A). $(I, K) s E^{2}-D s r c 41^{K R}$ heterozygous for $s E^{2}-D s r c 41^{+}$insertions (I) or Ras $1^{e 2 F}(K)$. (L) $s E^{2}-D s r c 41^{K R}$ heterozygote hemizygous for $\operatorname{sev}^{d 2}$. $|M|$ Double heterozygote for $s E^{2}-D s r c 41^{K R}$ insertion and a Dsrc41 deficiency. (N) Heterozygote for $s E^{2}-$ Dsrc $41^{K R Y F}$ insertion (KRYF-A).

morphological change in eye structure was detected in any of three transgenic lines with $s E^{2}-D s r c 41^{+}$(Fig. 4C). This finding is important, as under similar conditions, flies with a single copy of the wild-type Dsrc64 transgene showed extensively deformed eye morphology (Kussick et al. 19931.

Dsrc $41^{+}$and Dsrc $41^{Y F}$ transgenes were overexpressed in embryos by heat shock at $37^{\circ} \mathrm{C}$ for $30 \mathrm{~min}$. Overexpressed $D s r c 41^{+}$gave no appreciable effect on viability, whereas the overexpression of $D s r c 41^{Y F}$ was deleterious. 
Table 1. sE-Dsrc41 ${ }^{\mathrm{YF}}$ phenotype is affected by mutations in the sev signaling pathway

\begin{tabular}{|c|c|c|}
\hline Genotype & $\begin{array}{l}\text { Average number of } \\
\text { R7-type neurons }\end{array}$ & $\begin{array}{l}\text { Number of } \\
\text { ommatidia }\end{array}$ \\
\hline Wild type & $1.0 \pm 0.0\{ \pm 0.0\}$ & 234 \\
\hline WT-A & $1.0 \pm 0.0\{ \pm 0.1\}$ & 185 \\
\hline YF-A & $2.7 \pm 0.1( \pm 1.2)$ & 362 \\
\hline$d r k /+;$ YF-A & $1.0 \pm 0.01 \pm 0.21$ & 250 \\
\hline$E(\mathrm{sev}) 1 A^{e O P} /+$; YF-A & $1.0 \pm 0.0( \pm 0.0)$ & 200 \\
\hline Sos ${ }^{\times 122} /+;$ YF-A & $1.1 \pm 0.0( \pm 0.4)$ & 398 \\
\hline $\mathrm{YF}-\mathrm{A} / \operatorname{Ras} 1^{\mathrm{ezF}}$ & $1.1 \pm 0.1( \pm 0.4)$ & 387 \\
\hline$D-r a f^{1} /+$ YF-A & $1.3 \pm 0.1 i \pm 0.51$ & 267 \\
\hline Dsor $1^{n 1} /+;$ YF-A & $1.6 \pm 0.1( \pm 0.8\}$ & 228 \\
\hline YF-A; boss $1 /+$ & $1.9 \pm 0.1( \pm 0.8)$ & 340 \\
\hline YF-B & $2.1 \pm 0.1( \pm 0.9)$ & 350 \\
\hline YF-B; $b o s s^{7} / b o s s^{1}$ & $0.6 \pm 0.0( \pm 0.7)$ & 367 \\
\hline $\operatorname{sev}^{d 2} /+; \mathrm{YF}-\mathrm{A}$ & $1.4 \pm 0.1( \pm 0.6)$ & 309 \\
\hline$f l b^{3 C 81} /+;$ YF-A & $1.4 \pm 0.2( \pm 0.6)$ & 267 \\
\hline $\mathrm{YF}-\mathrm{A} / E(\mathrm{sev}) 3 \mathrm{~B}^{e l E}$ & $2.4 \pm 0.2( \pm 0.8)$ & 284 \\
\hline YF-A $/ E(s e v) 3 D^{e O Q}$ & $2.6 \pm 0.1( \pm 0.8)$ & 200 \\
\hline
\end{tabular}

For YF-A series, the transgene $\left(s E-D s r c 41^{Y F}\right)$ is heterozygous, whereas it is hemizygous in the case of YF-B. Two kinds of deviations are shown: The first one was calculated using the total number of eyes examined, and the second one, that of ommatidia. In the third column, only the total number of ommatidia examined is shown.

Close inspection of embryos homozygous for $D s r c 41^{\gamma F}$, heat-shocked at 4-7 hr of development, and cultured at $25^{\circ} \mathrm{C}$ until $13-16 \mathrm{hr}$ of development demonstrated that the overexpression of the activated Dsrc41 results in the failure of CNS shortening and dorsal closure, leading to embryonic lethality (Fig. 3J,K). In contrast, the overexpression of wild-type Dsrc64, along with that of its activated version, has been shown to be embryonic lethal (Kussick et al. 1993). It may thus follow that like vertebrate c-Src, Dsrc4l activity is repressed by stoichiometric phosphorylation of the carboxy-terminal conserved tyrosine residue (Tyr-511).

\section{Wild-type Dsrc4l is essential for normal eye morphogenesis}

c-Src with an inactive ATP-binding site because of a Lys $\rightarrow$ Arg substitution has been shown to be capable of serving as an effective antagonist of wild-type c-Src and, hence, to exhibit Src kinase-negative phenotypes/Chang et al. 1995). Thus, by P-mediated transformation (Fig. 2D), four transgenic fly lines (KR-A, KR-B, KR-C, and KR-D| were made that expressed a dominant-negative version of Dsrc41 $\left(D_{s r c 41}{ }^{K R}\right)$ driven by the $s E^{2}$ enhancer.

All four transgenic fly lines exhibited similar rough eye phenotypes (Fig. 4D). Cobalt sulfide staining (Fig. 6C) showed the shape and distribution of preclusters to be considerably affected. Either R3 or R4 precursors, or both, appeared to stain more weakly than their wild-type counterparts. As shown below, rhodamine-phalloidin and anti-DE-cadherin staining suggested that putative adherens junctions situated between R3 and R4 precursors is disorganized extensively in Dsrc $41^{K R}$ flies.

$\mathrm{H} 214$ is an enhancer-trap marker used to identify precursors for extra R7-type neurons (Mlodzik et al. 1992). The ectopic expression of $\mathrm{H} 214$ first became discernible in a very small fraction of late $D s r c 41^{K R}$ larvae (rows 17 and more posterior; Fig. $6 \mathrm{H}$ ), and ommatidia having extra $\mathrm{H} 214$-positive cells increased in number extensively during pupal stages (Fig. 6L). This suggests that the formation of ectopic pro-R7s in Dsrc $41^{K R}$ flies initiates at a stage much later than that for the formation of authentic R7 precursors.

During pupal stages, stacking of nuclei of two sevenup |svp|-positive cells became more apparent, and loss of svp-positive cells was observed in a small fraction of pupal ommatidia (Fig. 6J). In Dsrc41 ${ }^{K R}$ adult eyes, rhabdomere patterns and ommatidial spacing were highly abnormal: Most ommatidia contained one or two additional R7-type neurons, whereas one or a few outer photoreceptors were absent from $50 \%$ ommatidia (Figs. $5 \mathrm{I}$ and 7$\}$. Taken together, these results indicate that the dominant-negative form of Dsrc41 affects many steps of eye morphogenesis.

If, as expected, Dsrc41 ${ }^{\mathrm{KR}}$ serves as an antagonist of wild-type Dsrc41, eye phenotypes induced by the Dsrc4 $1^{K R}$ transgene should be suppressed and enhanced, respectively, by the increment and decrement of the dose of wild-type Dsrc41. Figures 4, E and F, 5, J and M, 6, B and $D$, and 7 show this to be the case. All Dsrc $41^{k R}$. dependent phenotypes examined so far were suppressed and enhanced, respectively, by simultaneous expression of an additional copy of a transgene, $s E^{2}-D s r C 41^{+}$and one-copy reduction of the endogenous Dsrc41 (Figs. 4E,F, $5 \mathrm{~J}, \mathrm{M}$, and $6 \mathrm{~B}, \mathrm{D})$. Note that the additional introduction of a heterozygous deficiency of the Dsrc41 locus results in the absence of R3/R4 photoreceptors stained strongly by cobalt sulfide (Fig. $6 \mathrm{D}$ ).

The Dsrc4 $1^{K R}$ transgene might function only to titrate out a negative regulator (such as Csk) to increase the fraction of the active, unphosphorylated form of endogeous (wild-type) Dsrc41. Activated Dsrc4l thus generated might undergo relocation to bind to target molecules (Kaplan et al. 1994) to consequently induce gainof-function phenotypes similar to those observed in Dsrc4 $1^{Y F}$ flies. But this appeared unlikely, as $|1|$ timing of the appearance of $D s r c 41^{K R}$-dependent defects differed considerably from that of Dsrc $4 l^{Y F}$-dependent defects, and (2) Dsrc41 ${ }^{K R}$ phenotypes were considerably enhanced by an additional YF mutation that may neutralize kinase activation by stimulating competitive inhibition upon target binding (Fig. 2D); see Fig. 5I,N). Dsrc41 may thus be essential for normal eye morphogenesis.

\section{boss-dependent, extra R7 formation in Dsrc41 ${ }^{\mathrm{YF}}$ ommatidia}

R7 formation in the wild type depends on Sev kinase activated by Boss expressed on the surface of $R 8$ (Kramer et al. 1991). Although R7 and cone cell precursors express $\mathrm{Sev}$, only pro- $\mathrm{R} 7 \mathrm{~s}$ are capable of coming into con- 
tact with R8 and receiving Boss signals (Tomlinson et al. 1987). This R7-R8 interaction may be demonstrated by genetic analysis along with Boss internalization (Reinke and Zipursky 1988; Kramer et al. 1991).

Table 1 and Figure 5, G and $\mathrm{H}$, show that the number of extra R7-type neurons in Dsrc $41^{Y F}$ flies decreases considerably with the one-dose reduction of boss or sev. This is of interest, as no effect of boss on gain-of-function mutations of Sev pathway genes has been reported (Basler et al. 1991; Fortini et al. 1992). Also, as in the case of the authentic pro-R7 (Fig. 6E), Boss was found to be internalized in a small but appreciable fraction of Dsrc $41^{Y F}$ ommatidial cells (Fig. $6 \mathrm{~F}$ ), which are most likely to be precursors for extra R7-type neurons. Our preliminary experiment also indicated a one-dose reduction of Dsrc41 to give no appreciable effect on $\mathrm{Sev}^{\mathrm{S} 11}$ (data not shown), suggesting that Dsrc4l functions upstream of Sev. $s E-D s r c 41^{Y F}$ would thus appear to endow cone cell precursors with the ability to make contact with R8 so as to receive the Boss signals for neuralization.

\section{Requirement of the Ras1/MAPK signal transduction pathway beginning with Boss for both gain-of-function and dominant-negative phenotypes of Dsrc41}

Genetic analysis using haploinsufficiency showed that the entire Rasl/mitogen-activated protein kinase (MAPK) signal transduction pathway is essential for Dsrc $41^{Y F}$-dependent formation of $\mathrm{R} 7$-type neurons $(\mathrm{Ta}$ ble 1); one-copy reduction of endogenous $d r k$, Sos, or
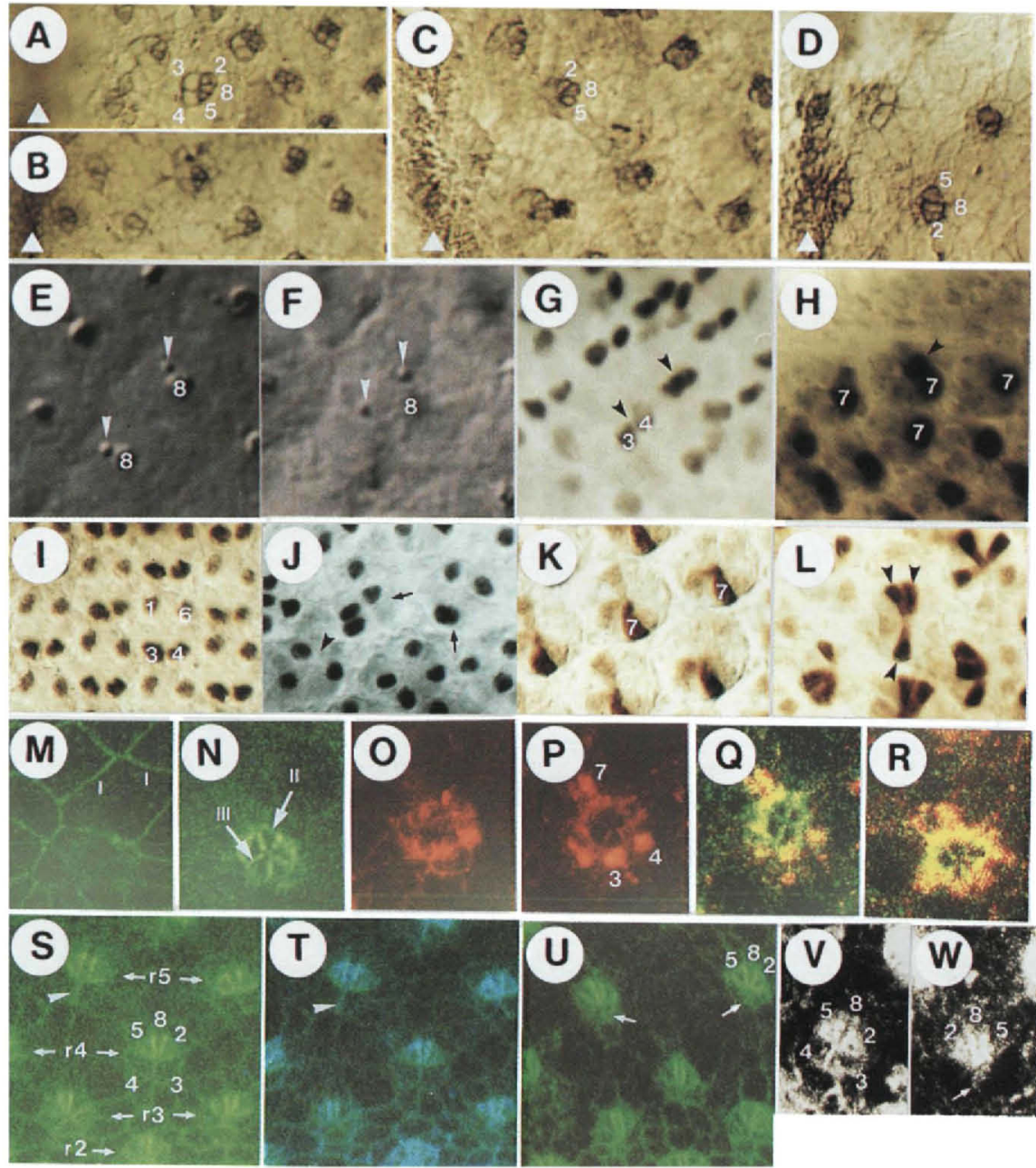

Figure 6. $(A-D)$ Cobalt sulfide staining of eye discs. (White triangles) Morphogenetic furrows. $(A)$ Wild type; $(B)$ double heterozygote for $s E^{2}-D s r c 41^{K R}$ and $s E^{2}-$ Dsrc $41^{+}$insertions. $(C)$ Heterozygote for $s E^{2}-D s r c 41^{K R}$ insertion (KR-A); (D) Double heterozygote for $s E^{2}-D s r c 41^{K R}$ insertion (KR-A) and a Dsrc41 deficiency. $(E)$ Boss internalization in wild-type pro-R7 (see arrowheads). $(F)$ Boss internalization in homozygous eyes for $s E-D s r c 41^{Y F}$ insertion (YF-A). (Arrowheads) Boss incorporated into authentic and ectopic pro-R7s adjacent to the same R8. $(G)$ svp-lacZ expression in larval eye discs heterozygous for $s E^{2}-D s r c 41^{K R}$ insertion. Arrowheads show irregularity in spacing between R3/ 4. (H) $H 214$ expression in a $s E^{2}-D s r c 41^{K R}$ transformant eye disc. (Arrowhead) Ectopic signals in cone cell precursors at the most matured stage. $(I, I)$ svp-lac $Z$ expression in pupal eye discs of wild-type $(I)$ and in a $s E^{2}-D s r c 41^{K R}$ transformant line $(J)$. (Arrows) Irregularity in spacing; (arrowhead) ommatidium containing only three svp-positive cells. $\{K, L\} H 214$ expression in pupal eye discs. $|K|$ Normal expression in wild-type pro-R7. $(L)$ Ectopic expression in $s E^{2}-D s r c 41^{K R}$ transformant line (see arrowheads). $(M-W)$ Confocal images. $(M, N, S, U)$ Anti-DE-cadherin antibody staining. $(O, P)$ Anti-LacZ antibody staining. $(Q, R)$ Double staining with anti$D E$-cadherin (green) and anti-LacZ (red) antibodies. $(T)$ Double staining with anti$D E$-cadherin antibody (green) and rhodamine-phalloidin (dark blue). $(V, W)$ Rhodamine-phalloidin staining. $(M, N) D E$-cadherin signals from the apical-most region and from the region 1-2 $\mu \mathrm{m}$ beneath the

apical surface of the same row 8 sample, respectively. (I-III) Type I to type III signals. (O) Apical-most LacZ signals. $(P \mid$ LacZ signals from the region $1-2 \mu \mathrm{m}$ beneath the apical surface. $(O, P)$ Signals from the same sample in row $9 .(Q, R)$ Row 9 and 10 signals at the 1-2 $\mu \mathrm{m}$ level. Yellow indicates colocalization of cadherin and the Dsrc4l-LacZ fusion protein. $(S-W)$ Staining patterns near the morphogenetic furrow. (Arrowheads) Strong cadherin signals along row 5 R3-R4 boundaries. Light blue in $T$ indicates colocalization of cadherin and actin fibers. The absence of cadherin-positive, putative adherens junctions $(U)$ and actin cytoskeleton $(W)$ along boundaries between row $5 \mathrm{R} 3 / \mathrm{R} 4$ expressing $D s r c 41^{K R}$ is indicated by arrows. 
Takahashi et al.

Ras1 virtually completely suppresses ectopic neuralization. Enhanced effects of these elements may be partly attributable to the fact that not only the Boss/Sev signal but also the DER (Drosophila epidermal growth factor (EGF) receptor] signal contributes to neuralization in the $\bar{D}_{S r C 4} \overline{1}^{Y F}$ mutant (Table 1). However, unlike the Sev pathway in the authentic R7 (Simon et al. 1991), cdc37 and $E / \mathrm{sev} / 3 D$ may not be required for ectopic neuralization in the Dsrc41 ${ }^{Y F}$ mutant.

Dominant-negative phenotypes of Dsrc41 also depend on the Rasl/MAPK signal transduction pathway beginning with Boss \{Figs. 5 and 7\}. Lost outer photoreceptors were virtually completely recovered by one-copy reduction of either Ras1 or boss or the absence of sev. The formation of extra R7-type neurons was also partially reversed (Figs. 5 and 7). However, it should be noted here that the Boss protein expressed in R8 may not be related to these phenotypes because neuralization of extra R7like cells and loss of outer photoreceptors in Dsrc $41^{K R}$ flies occur mainly during pupal stages when Boss expression is not specific to R8 but rather ubiquitous and nonfunctional (Kramer et al. 1991).

Localization of Dsrc41 ${ }^{Y F_{-}}$LacZ in the close vicinity of $\mathrm{DE}$-cadherin-positive, putative adherens iunctions

In vertebrate cultured cells, Src is relocalized to focal adhesions following activation and controls cytoskeleton organization and cell signaling (Kaplan et al. 1994). Although no focal adhesions have been recognized in larval eyes, larval ommatidial cells are connected by adherens iunctions (Longley and Ready 1995), which are presumably functional analogs of focal adhesions in the cell-cell adhesion system (Gumbiner 1996). In vertebrates, classic cadherins such as E-cadherin have been shown to be essential not only for formation of adherens junctions but also for the maintenance of cell-cell contacts and actin cytoskeleton (Takeichi 1991; Gumbiner 1996). Thus, we first examined the spatial distribution of DE-cadherin, a putative ortholog in Drosophila of vertebrate E-cadherin (Oda et al. 1994), and compared it with that of the Dsrc4 $1^{\mathrm{YF}}-\mathrm{LacZ}$ fusion protein.

Figure $6 \mathrm{M}$ shows the distribution of $D \mathrm{E}$-cadherin signals obtained by confocal microscopy, indicating that $D E$-cadherin distributes widely along boundaries of many ommatidial and nonommatidial cells. Because the distribution of actin fibers /cytoskeleton! visualized by rhodamine-phalloidin was very similar, if not identical, to that of $D E$-cadherin (Fig. $6 \mathrm{~T}$ ), $D \mathrm{E}$-cadherin-positive regions found in larval eyes would appear to correspond to adherens junctions and/or their close relatives in developing eyes.

In normal eye development, ommatidial formation is
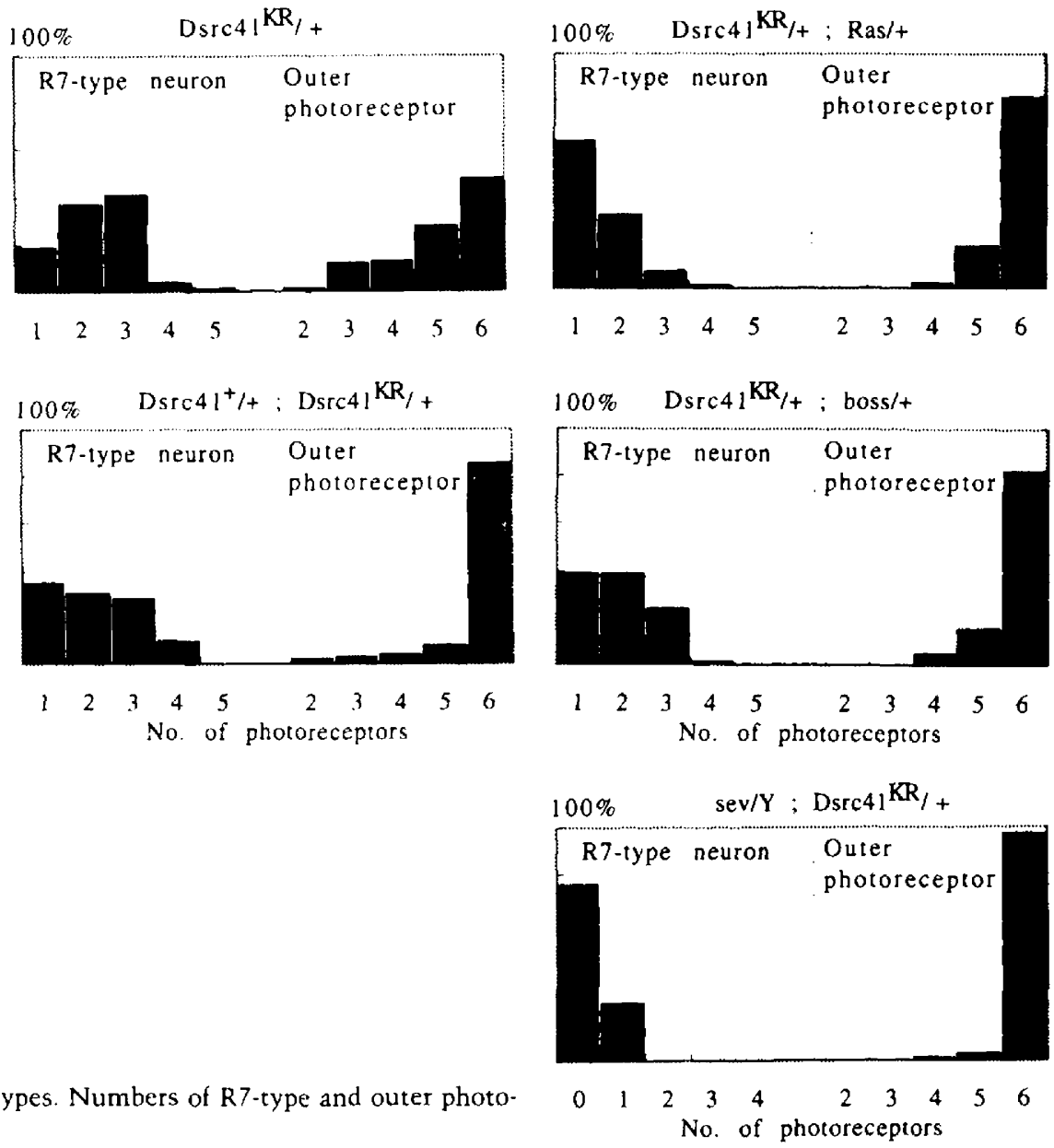

Figure 7. Suppression of Dsrc41 $1^{K R}$ phenotypes. Numbers of R7-type and outer photoreceptors per ommatidium are indicated. 
lineage independent, and each ommatidial precursor is assembled through the stepwise recruitment of cells into stereotyped clusters (for review, see Wolff and Ready 1993|. Developing photoreceptors express neural antigen in a fixed sequence: $R 8$ expresses neural antigens first and is followed by the R2/R5 pair and then by the R3/R4 pair. Interestingly, as shown in Figure 6S, the order and timing of the appearance of strong cadherin signals were virtually identical to those of neural antigens (for neural antigen expression, see Wolff and Ready 1993). Strong cadherin signals first appeared along boundaries of $\mathrm{R} 8$ in row 2. Photoreceptors R2 and R5 begin expressing strong cadherin signals in rows 3 and 4 . Strong cadherin signals appeared along $\mathrm{R} 3-\mathrm{R} 4$ boundaries in row 5 . It may thus follow that the formation of adherens junctions is initiated at a very early stage of photoreceptor development.

In row 9 or 10 , three distinct types of $D E$-cadherin signals were recognized. Type I signals distributed along the apical-most boundaries of cells not yet recruited into ommatidia and putative cone cell precursors (Fig. 6M). Type Il signals $(0.5-2 \mu \mathrm{m}$ beneath the apical surface) were found along boundaries between cone cells and photoreceptors (Fig. 6N). Type III signals, $\sim 1 \mu \mathrm{m}$ in height and situated at $\sim 1.5 \mu \mathrm{m}$ beneath the apical surface, were readily apparent along boundaries between neighboring photoreceptor precursors (Fig. $6 \mathrm{~N}$ ). Locations of type II and type III signals appeared to coincide with those of zonula adherens visualized by electron microscopy (Longley and Ready 1995), suggesting that type II and type III signals may correspond to zonula adherens. Recently, this notion was confirmed further in an independent experiment ( $T$. Uemura and $H$. Oda, pers. comm.).

The distribution of the fusion protein consisting of activated Dsrc4l and LacZ $\left\{s E^{2}-D s r c 41^{Y F}\right] a c Z$; Fig. 2D) was examined using anti-LacZ for Escherichia coli $\beta$-galactosidase antibody. $s E^{2}-D s r c 41^{Y F}-l a c Z$ flies exhibited eyes slightly rough in appearance. Confocal images of anti-LacZ and anti-DE-cadherin antibody double staining gave strong indication that Dsrc41-LacZ signals considerably overlap three types of $D E$-cadherin signals (Fig. 6R). However, it should be noted that type II signals of Dsrc41-LacZ exhibit marked apical extension |Fig. 6O), and the appearance of type III signals of $D E$-cadherin precedes those of Dsrc41-LacZ (Fig. 6, cf. Q and R). Taken together, these results suggest that activated Dsrc4l is localized in close proximity to $D E$-cadherin-positive adherens junctions and/or other types of DE-cadherin aggregates.

\section{Failure in formation of stable putative adherens junctions between adjacent precluster cells expressing Dsrc41 ${ }^{\mathrm{KR}}$}

As shown in Figure 6C, a transient expression of Dsrc $41^{K R}$ in precursors for $\mathrm{R} 3$ and $\mathrm{R} 4$ resulted in ommatidial disorders in preclusters. To examine whether these defects are related to possible alterations in cytoskelton organization and/or cell-cell contacts, the distribution of $D \mathrm{E}$-cadherin and actin fibers in $D s r c 41^{K R}$ preclusters was examined. As shown in Figure 6, U and W, little or no signal for actin fibers and DE-cadherin could be de- tected along boundaries between $\mathrm{R} 3$ and $\mathrm{R} 4$ precursors. $D E$-cadherin and actin fiber signals along $R 3 / R 2$ and $R 4 /$ R5 boundaries decreased considerably in intensity. Therefore, it may follow that the reduction of Dsrc4l kinase activity results in disorganization of cytoskeletal actin fibers and putative adherens junctions containing $D E$-cadherin, which is presumed to be responsible for cell-cell contacts (Gumbiner 1996).

\section{Discussion}

The protein encoded by Dsrc41 shows hallmarks of a bona fide Src family member. Our results show that Dsrc41 is a much closer relative of vertebrate c-src than is Dsrc64, long considered to be a unique gene belonging to the src family in Drosophila (Kussick et al. 1993). The phylogenetic tree in Figure $2 \mathrm{C}$ suggests that Dsrc64 forms its own group distantly related to vertebrate src family members and Dsrc41. Kussick et al. (1993) showed dominant-negative and gain-of-function mutations of Dsrc64 to cause severe eye defects. As in the case of Dsrc41, Dsrc64 gain-of-function phenotypes are partially suppressed by reducing Ras-pathway signals, and thus DsrC64 may be considered to have functions similar to those of Dsrc41. Eye defects in Dsrc41 gain-of-function mutants, however, are much less severe compared to those in Dsrc64 mutants, and unlike wild-type Dsrc64, the overexpression of wild-type Dsrc41 is not attended with any appreciable phenotype (Fig. $4 \mathrm{C}$ ). These results and apparent differences in expression for $D$ src64 and Dsrc41 suggest that Dsrc64 and Dsrc41 are src relatives in Drosophila, each with different roles in cell differentiation and/or proliferation.

\section{Is Dsrc41 required for control of cytoskeleton organization and cell-cell contacts?}

In vertebrates, classic cadherins, including E-cadherin, are localized in zonula adherens or intercellular adherens junctions and are required to regulate cell-cell contact and tissue adhesion (Takeichi 1991). Carboxy-terminal portions of the cytoplasmic domain are essential for interaction with the cytoskeleton (actin fibers) and for cadherin-adhesive activity (Nagafuchi and Takeichi 1988). $D E$-cadherin shares in common many properties with vertebrate classic cadherins and is presumed to be a homolog of Drosophila (Oda et al. 1994). These investigators showed $D E$-cadherin to be present in zonula adherens in the case of embryos.

Our results showed $D E$-cadherin type II and type III signals to be located at $\sim 1 \mu \mathrm{m}$ beneath the apical surface of larval ommatidial cells, where zonula adherens junctions have been recognized by electron microscopy (Longley and Ready 1995). Thus, DE-cadherin-positive type II and type III signals are quite likely to correspond to adherens junctions in developing photoreceptor cells. Rhodamine-phalloidin staining showed that most signals for actin fibers colocalize with three types of $D E$ cadherin signals (Fig. 6T). Because classic cadherins constitute the actin-based zonula adherens (Takeichi 1991) in vertebrates, not only type II and type III, but also type 
I, cadherin signals may be involved in regulation of cellcell contacts and cytoskeleton organization.

By the immunostaining of activated Dsrc4l with a LacZ tag, the activated Dsrc41-LacZ fusion protein was shown predominantly to be located quite close to putative adherens junctions containing cadherin (Fig. 6Q). A transient expression of the dominant-negative form of Dsrc4l in preclusters was noted to cause failure at putative adherens junctions, not only in the formation or maintenance of the actin cytoskeleton but also in the deposit of DE-cadherin (Fig. 6U). Cobalt sulfide staining showed the differentiation of R3 and R4 to be affected by Dsrc $41^{K R}$ expression (Fig. 6C). This effect was enhanced considerably by one-copy reduction of the endogenous wild-type Dsrc41 gene (Fig. 6D). Thus, these results suggest that complexes consisting of Dsrc4l, actin, and $D E$. cadherin and/or their localized concerted functions are essential for the formation and/or maintenance of cytoskeleton structures and cell-cell contacts, at least in precluster cells.

The disappearance of actin fibers and putative adherens junctions in precluster cells was observed only in dominant-negative mutants. Figure $6 \mathrm{~T}$ shows the presence of actin fibers along with $D E$-cadherin in all precluster cells in wild-type and gain-of-function mutants. These findings cannot be explained by kinase-independent, amino-terminal activity of $\mathrm{Src}$ as proposed by $\mathrm{Ka}$ plan et al. (1994). The kinase activity of wild-type Dsrc4 1 may thus be considered essential for the formation and/ or maintenance of the cytoskeleton and adherens junc. tions in precluster cells.

In more mature ommatidia, excess tyrosine kinase activity of Dsrc41 may affect cell-cell contact; as Figure 6F suggests, extra $R 7$ cell formation in gain-of-function mutants of Dstc41 is partially dependent on ectopic contact between Boss-expressing R8 and cone cell precursors, which express Sev but cannot make contact with R8 during wild-type development.

Kaplan et al. (1994) showed the dephosphorylation of conserved carboxy-terminal tyrosine of c-Src to cause redistribution of c-Src to focal adhesions and to stimulate the Src-dependent phosphorylation of various cytoskeletal proteins including focal adhesion kinase (FAK). Fibronectin binding to integrins on NIH-3T3 fibroblasts promotes association among c-Src, Grb2 (I)rk), and FAK, and the formation of an integrin-activated signaling complex linked to the Ras/MAPK signal transduction pathway (Schlaepfer et al. 1994). Analysis of mouse embryos lacking Csk demonstrated the importance of the dephosphorylation of Src family kinases in the regulation of embryonic cytoskeleton organization (Thomas et al. 1995). These findings and the present results for Drosophila suggest Src to be critically essential to the control of cell morphology and cell-cell contact in vertebrate and invertebrate development.

\section{A part of the Ras1/MAPK signal may originate from Dsrc41 itself}

Table 1 shows not only Sev but also DER to be involved in $D_{s r C 4}{ }^{Y F}$-dependent neuralization. Vertebrate EGF re- ceptor tyrosine kinase has been shown to be activated via association with v-Src (Maa et al. 1995). Therefore, similarly to the vertebrate EGF receptor, might be activated by $D s r c 41^{Y F}$, the activated version of Dsrc4l. In vertebrate cultured cells, $v$-Src, or the activated version of Src, is localized to focal adhesion contacts to phosphorylate FAK and/or p62, whose phosphorylation provides binding sites for Grb2 (Drk) and/or Sos (Richard et al. 19951. Our results indicate (activated) Dsrc4l to be localized in putative adherens junctions along with more apical regions. Thus, it is also possible that a part of the Ras signal for neuralization may originate from the activated Dsrc4l itself.

\section{Materials and methods}

plasmids

pYCl.8, a P vector with a vermilion marker, was obtained from L. Searles (Fridell and Searles 1991). P plasmids expressing Dsrc41 with or without mutations were made as follows. The hsp 70 promoter and polyadenylation signal, respectively, were introduced into the $\mathrm{Xbal}$ and Hpal-Notl sites of pYC1.8M, a derivative of $\mathrm{pYCl} .8$ with multicloning sites $(\mathrm{F}$. Takahashi, unpubl. 1 to generate pVH9. Then, a 1.8 -kb Scal-KpnI fragment of Dsrc4l cDNA with or without mutations (see below) was introduced into the BgllI-Hpal site of pVH9. Finally, one or two copies of a $670 \cdot \mathrm{bp}$ sev enhancer (Bowtell et al. 1991) were introduced between the Sall and Smal sites just upstream of the Dstc41 minigene. $s E-D S r C 41^{Y F}-l a c Z$ was made similarly. The lacZ fragment was introduced into the $B a m H I$ site of Bluescript to generate pZSK-R. Dsrc41 ${ }^{\mathrm{YF}}$ was inserted into the KpnI-PstI site of pZSK-R. The resultant Dsrc41 ${ }^{\mathrm{YF}}-\mathrm{lacZ}$ gene was introduced into pVH9, gencrating $s E^{2}$.

\section{Isolation of genomic DNA and CDNA clones}

Genomic DNA and pupal cDNA clones of Dsrc41 were isolated from libraries described previously (Kojima et al. 1991). For isolation of the first genomic DNA clone $(\lambda g f k 1)$, dt $k 5$ (Shishido et al. 1991) was used as a probe. Hybridization was carried out at $55^{\circ} \mathrm{C}$ for $12 \mathrm{hr}$ in $\mathrm{H}$ buffer $[0.1 \mathrm{M}$ Tris- $\mathrm{HCl}$ ipH 7.5 l containing 1 $M \mathrm{NaCl}, 10 \mathrm{~mm}$ EDTA, $10 \times$ Denhardt's solution, $0.2 \% \mathrm{SDS}$, and $200 \mu \mathrm{g} / \mathrm{ml}$ of heat-denatured salmon sperm DNA|, which includes ${ }^{32} \mathrm{P}$-labeled probe DNA. Filters were washed at $55^{\circ} \mathrm{C}$ for $1-2 \mathrm{hr}$ in $2 \times$ SSC $(15 \mathrm{~mm}$ sodium citrate with $150 \mathrm{~mm} \mathrm{NaCl})$ containing $0.1 \%$ SDS. The second genomic DNA clone ( $\lambda g f k 2)$ was isolated using the cDNA insert of $\lambda c f k$ (see belowl as a probe. cDNA clones were isolated using a $0.9 \cdot \mathrm{kb}$ HindIII-KpnI fragment of $\lambda g f k 1$ (see line $A$ in Fig. $1 \mathrm{~A}$ ) as a probe. Hybridization was performed at $65^{\circ} \mathrm{C}$ for $12 \mathrm{hr}$ in $\mathrm{H}$ buffer, including ${ }^{32} \mathrm{P}$-labeled, heat-denatured probe DNA. Filters were washed at $65^{\circ} \mathrm{C}$ tor $1-2 \mathrm{hr}$ in $0.1 \times$ SSC with $0.1 \%$ SDS

\section{Southern and Northern blotting}

Canton-S DNA digested with suitable restriction enzymes was subjected to Southern analysis under less stringent conditions using a 0.9 -kb HindIII-KpnI fragment of $\lambda g f k 1$ as a probe. Filters were hybridized at $60^{\circ} \mathrm{C}$ in $\mathrm{H}$ buffer for $24 \mathrm{hr}$ and then washed at $60^{\circ} \mathrm{C}$ in $2 \times$ SSC with $0.1 \%$ SDS for $1-2 \mathrm{hr}$. Northern hybridization was carried out as described previously (Kojima et al. 19911.

\section{In vitro mutagenesis}

Tyrosine 511 of Dsrc4l was replaced by a phenylalanine residue using PCR, in which one of the primers contained a mutated 
sequence. With the introduction of the YF mutant, the following primers were used: 5 '-GGCGCTAGCAGGCGCCAGATT. TCCC and 5'-AGTA-GGCCTGCGCCTCTTTGAAGTC junderline, mutated basel. The KR mutant was generated by the Kunkel method (Sambrook et al. 1989) using 5'-TTCAGAGT TCTAATTGCCAC as the antisense primer. All other procedures for molecular cloning were as described by Sambrook et al. (1989).

\section{Germ-line transformation}

A mixture of $300 \mu \mathrm{g} / \mathrm{ml}$ of Dsrc 41 plasmid DNA and $150 \mu \mathrm{g} / \mathrm{ml}$ of a helper plasmid, pUChs $\Delta 2-3$, was injected into $v^{360} ; 5 y^{506}$ embryos (Spradling and Rubin 1982). Three (WT-A (first chromosomel, B (third), and C (first)] and two [YF-A (third) and B (first)| viable transformant lines having wild-type and activated Dsrc41 minigenes, respectively, were obtained. In the case of Dsrc41 ${ }^{K R}$, and Dsrc $41^{Y F}-l a c Z$, four and two transformant lines [KR-A (second), B (second), C (third), and D (third); DL-A (second) and $B$ (second)| were obtained. In addition, two DsrC41 KRYF lines |KRYF-A and KRYF-B| were recovered.

\section{Fly stocks}

Canton-S (wild type) and $v^{36 t}{ }_{i} y^{500}$ were our laboratory stocks, and $s v p^{H 162}$, an enhancer trap line of svp was obtained from G. Rubin |University of California, Berkeley|. A fly strain having a Dsrc41 deficiency $\left[\ln (2 R) b w^{V D e 2 L} C y^{R} / G l a\right]$ and $f l b^{3 C .81}$, a DER mutant, respectively, were from Umeà and Tübingen stock centers. The following were used as crosses to examine suppressor/ enhancer activity: y D-raf'/Binsc, oc ptg (Tsuda et al. 1993); y w spl sn Dsor $1^{r 1} /$ Binsc, oc ptg (Tsuda et al. 1993); Sos ${ }^{\times 122} / C y O$ (Rogge et al. 1991), y E(sev) $1 A^{\text {eOP' }} /$ Binsc,oc ptg (Simon et al. 1991); $E(\mathrm{sev}) 2 B^{e O A}$ (drk)/CyO (Simon ct al. 1991); E(sev) $3 B^{e t 1}$. TM6B, Tb (Simon et al. 1991); Ras 1 ${ }^{e 2 F} / T M 6 B$, Tb iSimon et al. 1991); $E(\mathrm{sev}) 3 D^{c 0 Q} / \mathrm{TM} 6 B$, Tb (Simon et al. 1991); sev d? (Simon et al. 1991); and boss ${ }^{2} h^{2} r^{500}$ (Kramer et al. 1991). Enhancer trap lines $H 214$ and $A E 127$ (svp) were obtained from Y. Hiromi (Princeton University, NI).

\section{Immunohistochemistry and in situ hybridization}

Immunostaining and in situ hybridization were performed essentially according to Higashijima et al. $11992 \mid$ and Shishido et al. (1993). Origins of anti-HRP, anti-LacZ $(E$. coli $\beta$-galactosidase) antibodies, and $\mathrm{mAb} 22 \mathrm{Cl} 10$ were described in Higashijima et al. (1992). Anti-Boss and anti-DE-cadherin antibody (DCAD2) were gifts of L. Zipursky (University of California, Los Angeles) and T. Uemura (Kyoto University, Japan). A 1.9-kb EcoRI-StuI fragment of Dsrc41 cDNA was used as a template for making RNA probe.

\section{Electron microscopy}

Samples for electron microscopy were prepared and observed as described previously (Koiima et al. 1991).

\section{Acknowledgments}

We thank E. Shishido and Y. Emori for many suggestions at the early stages of cloning. We also thank Y. Nishida, G. Rubin, L. Zipursky, U. Baneriee, E. Hafen, T. Uemura, and Y. Jan for fly stocks and antibodies. This work was supported in part by grants from The Ministry of Science, Culture, and Education of Japan and The New Energy and Industrial Technology Development Organization (to K.S).
The publication costs of this article were defrayed in part by payment of page charges. This article must therefore be hereby marked "advertisement" in accordance with 18 USC section 1734 solely to indicate this fact.

\section{References}

Basler, K., B. Christen, and E. Hafen. 1991. Ligand-independent activation of the sevenless receptor cyrosine kinase changes the tate of cells in the developing Drosophila eye. Cell 64: 1069-1081.

Bowtell, D.D.L., T. Lila, W.M. Michael, D. Hackett, and G.M. Rubin. 1991. Analysis of the enhancer element that controls expression of sevenless in the developing Drosophila eye. Proc. Natl. Acad. Sci. 88: 6853-6857.

Campos-Ortega, J.A. and V. Hartenstein. 1985. The embryonic development of Drosophila melanogaster. Springer-Verlag, Bcrlin, Germany.

Cartwright, C.A., W. Eckhart, S. Simon, and P.L. Kaplan. 1987. Cell transformation by $p$ p $60^{c} \cdots$ mutated in the carboxy-terminal regulatory domain. Cell 49:83-91.

Chang, J.-H., S. Gill, J. Settleman, and S.J. Parsons. 1995. c-Src regulates the simultaneous rearrangement of actin cytoskelcton. p190RhoGAP, and pl20RasGAP following epidermal growth factor stimulation. I. Cell Biol. 130: 355-368.

Cooper, I.A. and B Howell. 1993. The when and how of Src regulation. Cell 73: 1051-1054.

Cotton, P.C. and J.S. Brugge. 1983. Neural Tissues express high levels of the cellular src gene product pp60 6 src. Mol. Cell. Biol. 3: $1157-1162$.

Fortini, M.E., M.A. Simon, and G.M. Rubin. 1992. Signalling by the sevenless protein tyrosine kinase is mimicked by Ras 1 activation. Nature 355: 559-561.

Fridell, Y.-W.C. and L.L. Searles. 1991 Vermilion as a small selectable marker gene for Drosophila transformation. $\mathrm{Nu}$ cleic Acids Res. 19: 5082.

Fults, D.W., A.C. Towle, J.M. Lauder, and P.F. Maness. 1985. pp $60^{\circ} \times c$ in the developing cerebellum. Mol. Cell. Biol. 5: $27-32$.

Gregory, R.J., K.L. Kammermeyer, W.S. Vincent III, and S.G. Wadsworth. 1987. Primary sequence and developmental expression of a novel Drosophila melanogaster src gene. Mol. Cell Biol. 7: 2119-2127.

Gumbiner, B.M. 1996. Cell adhesion: The molecular basis of tissue architecture and morphogenesis. Cell 84: 345-357.

Hanks, S.K., A.M. Quinn, and T. Hunter. 1988. The protein kinase family: Conserved features and deduced phylogeny of the catalytic domains. Science 241: $42-52$.

Henkemeyer, M.J., R.L. Bennett, F.B. Gertler, and F.M. Hoffmann. 1988. DNA sequence, structure, and tyrosine kinase activity of the Drosophila melanogaster Abelson proto-oncogene homolog. Mol. Cell. Biol. 8: 843-853.

Higashijima, S., T. Kojima, T. Michiue, S. Ishimaru, Y. Emori, and K. Saigo. 1992. Dual Bar homeo box genes of Drosophila required in two photoreceptor cells, Rl and R6, and primary pigment cells for normal eye development. Genes \& Dev. 6: $50-60$.

Iba, H., F.R. Cross, E.A. Garber, and H. Hanafusa. 1985. Low level of cellular protein phosphorylation by nontransforming overproduced p60 ${ }^{\mathrm{c} s \mathrm{sc}}$. Mol. Cell. Biol. 5: 1058-1066.

Imamoto, A. and P. Soriano. 1993. Disruption of the csk gene, encoding a negative regulator of Src family tyrosine kinases, leads to neural tube defects and embryonic lethality in mice. Cell 73: $1117-1124$. 
Kaplan, K.B., K.B. Bibbins, J.R. Swedlow, M. Arnaud, D.O. Morgan, and H.E. Varmus. 1994. Association of the amino-terminal half of c-Src with focal adhesions alters their properties and is regulated by phosphorylation of tyrosine 527 . EMBO /. 13: 4745-4756.

Kojima, T., S. Ishimaru, S. Higashijima, E. Takayama, H. Akimaru, M. Sone, Y. Emori, and K. Saigo. 1991. Identification of a different type homeobox gene, $B a r H I$, possibly causing Bor $(B)$ and $O m(1 D)$ mutations in Drosophila. Proc. Natl. Acad. Sci. 88: 4343-4347.

Kramer, H., R.L. Cagan, and S.L. Zipursky. 1991. Interaction of bride of sevenless membrane-bound ligand and the sevenless tyrosine-kinase receptor. Nature 352: 207-212.

Kussick, S.J. and J.A. Cooper. 1992. Overexpressed Drosophila src $64 \mathrm{~B}$ is phosphorylated at its carboxy-terminal tyrosine, but is not catalytically repressed, in cultured Drosophila cells. Oncogene 7: 2461-2470.

Kussick, S.J., K. Basler, and I.A. Cooper. 1993. Ras1-dependent signaling by ectopically-expressed Drosophila src gene product in the embryo and developing eye. Oncogene 8: 27912803.

Longley, R.L. Ir. and D.F. Ready. 1995. Integrins and the development of three-dimensional structure in the Drosophila compound eye. Dev. Biol. 171: 415-433.

Maa, M.-C., T.-H. Leu, D.J. McCarley, R.C. Schatzman, and S.J. Parsons. 1995. Potentiation of epidermal growth factor receptor-mediated oncogenesis by c-Src: Implications for the etiology of multiple human cancers. Proc. Natl. Acad. Sci. 92: $6981-6985$.

Manness, P.F., L.K. Sorge, and D.W. Fults. 1986. An early developmental phase of pp60 ${ }^{c-s t c}$ expression in the neural ectoderm. Dev. Biol. 117: 83-89.

Mlodzik, M., Y. Hiromi, C.S. Goodman, and G.M. Rubin. 1992. The presumptive R7 cell of the developing Drosophila eye receives positional information independent of sevenless, boss and sina. Mech. Dev. 37: 37-42.

Molina, T.I., K. Kishihara, D.P. Siderovski, W. van Ewijk, A. Narendran, E. Timms, A. Wakeham, C.J. Paige, K.-U. Hartmann, A. Veillette, D. Davidson, and T.W. Mak. 1992. Profound block in thymocyte development in mice lacking p56 ich. Nature 357: 161-164.

Nada, S., T. Yagi, H. Takeda, T. Tokunaga, H. Nakagawa, Y. Ikawa, M. Okada, and S. Aizawa. 1993. Constitutive activation of Src family kinases in mouse embryos that lack Csk. Cell 73: 1125-1135.

Nagafuchi, A. and M. Takeichi. 1988. Cell binding function of $\mathrm{E}$-cadherin is regulated by the cytoplasmic domain. EMBO /. 7: $3679-3684$.

Oda, H., T. Uemura, Y. Harada, Y. Iwai, and M. Takeichi. 1994. A Drosophila homolog of cadherin associated with armadillo and essential for embryonic cell-cell adhesion. Dev. Biol. 165: 716-726.

Reinke, R. and S.L. Zipursky. 1988. Cell-cell interaction in the Drosophila retina: The bride of sevenless gene is required in photoreceptor cell R8 for R7 cell development. Cell 55: 321 330.

Richard, S., D. Yu, K.J. Blumer, D. Hausladen, M.W. Olszowy, P.A. Connelly, and A.S. Shaw. 1995. Association of p62, a multifunctional $\mathrm{SH} 2$ - and $\mathrm{SH} 3$-domain-binding protein, with sIc family tyrosine kinase, Grb2, and phospholipase Cy-1. Mol. Cell. Biol. 15: 186-197.

Rogge, R.D., C.A. Karlovich, and U. Banerjee. 1991. Genetic dissection of a neurodevelopmental pathway: Son of sevenless functions downstream of the sevenless and EGF receptor tyrosine kinases. Cell 64: 39-48.

Sambrook, I., E.F. Fritsch, and T. Maniatis. 1989. Molecular cloning: A laboratory manual. Cold Spring Harbor Laboratory Press, Cold Spring Harbor, NY.

Schlaepfer, D.D., S.K. Hanks, T. Hunter, and P. van der Geer. 1994. Integrin-mediated signal transduction linked to Ras pathway by GRB2 binding to focal adhesion kinase. Nature 372: 786-791.

Shalloway, D., P.M. Coussens, and P. Yaciuk. 1984. Overexpression of the c-src protein does not induce transformation of NIH 3T3 cells. Proc. Natl. Acad. Sci. 81: 7071-7075.

Shishido, E., Y. Emori, and K. Saigo. 1991. Identification of seven novel protein-tyrosine kinase genes of Drosophila by the polymerase chain reaction. FEBS Lett. 289: 235-238.

Shishido, E., S. Higashijima, Y. Emori, and K. Saigo. 1993. Two FGF-receptor homologues of Drosophila: One is expressed in mesodermal primordium in early embryos. Development 117: 751-761.

Simon, M.A., B. Drees, T. Kornberg, and J.M. Bishop. 1985. The nucleotide sequence and the tissue-specific expression of Drosophila c-src. Cell 42: 831-840.

Simon, M.A., D.D.L. Bowtell, G.S. Dodson, T.R. Laverty, and G.M. Rubin. 1991. Rasl and a putative guanine nucleotide exchange factor perform crucial steps in signaling by the sevenless protein tyrosine kinase. Cell 67: 701-716.

Spradling, A.C. and G.M. Rubin. 1982. Transposition of cloned P elements into Drosophila germ line chromosomes. Sci. ence 218: 341-347.

Stark, W.S. and R. Sapp. 1987. Ultrastructure of the retina of Drosophila melanogaster: The mutant ora louter rhabdomeres absentl and its inhibition of degeneration in $r d g B$ |retinal degeneration-B). /. Neurogenet. 4: 227-240.

Stein, P.L., H. Vogel, and P. Soriano. 1994. Combined deficiencies of Src, Fyn, and Yes tyrosine kinases in mutant mice. Genes \& Dev. 8: 1999-2007.

Takeichi, M. 1991. Cadherin cell adhesion receptors as a morphogenetic regulator. Science 251: 1451-1455.

Thomas, S.M., P. Soriano, and A. Imamoto. 1995. Specific and redundant roles of Src and Fyn in organizing the cytoskeleton. Nature 376: 267-271

Tomlinson, A., D.D.L. Bowtell, E. Hafen, and G.M. Rubin. 1987. Localization of the sevenless protein, a putative receptor for positional information, in the eye imaginal disc of Drosophila. Cell 51: 143-150.

Tsuda, L., Y.H. Inoue, M.-A. Yoo, M. Mizuno, M. Hata, Y.-M. Lim, T. Adachi-Yamada, H. Ryo, Y. Masamune, and Y. Nishida. 1993. A protein kinase similar to MAP kinase activator acts downstream of the Raf kinase in Drosophila. Cell 72: 407-414.

Tsukada, S., D.C. Saffran, D.J. Rawlings, O. Parolini, R.C. Allen, I. Klisak, R.S. Sparkes, H. Kubagawa, T. Mohandas, S. Quan, J.W. Belmont, M.D. Cooper, M.E. Conley, and O.N. Witte. 1993. Deficient expression of a B cell cytoplasmic tyrosine kinase in human $\mathrm{X}$-linked agmmaglobulinemia. Cell 72: 279-290.

Wolff, T. and D.F. Ready. 1993. Pattern formation in the Drosophila retina. In The development of Drosophila melanogaster \{ed. M. Bate and A.M. Arias\}, pp. 1277-1325. Cold Spring Harbor Laboratory Press, Cold Spring Harbor, NY.

Yagi, T., S. Aizawa, T. Tokunaga, Y. Shigetani, N. Takeda, and Y Ikawa. 1993. A role for Fyn tyrosine kinase in the sucking behaviour of neonatal mice. Nature 366: 742-745.

Zhao, Y.-H., J.G. Krueger, and M. Sudol. 1990. Expression of cellular-yes protein in mammalian tissues. Oncogene 5: 1629-1635. 


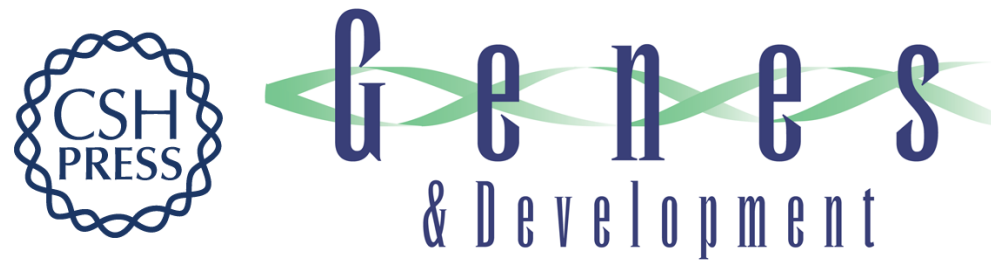

\section{Regulation of cell-cell contacts in developing Drosophila eyes by Dsrc41, a new, close relative of vertebrate c-src.}

F Takahashi, S Endo, T Kojima, et al.

Genes Dev. 1996, 10:

Access the most recent version at doi:10.1101/gad.10.13.1645

References This article cites 47 articles, 16 of which can be accessed free at:

http://genesdev.cshlp.org/content/10/13/1645.full.html\#ref-list-1

License

Email Alerting

Service

Receive free email alerts when new articles cite this article - sign up in the box at the top right corner of the article or click here.

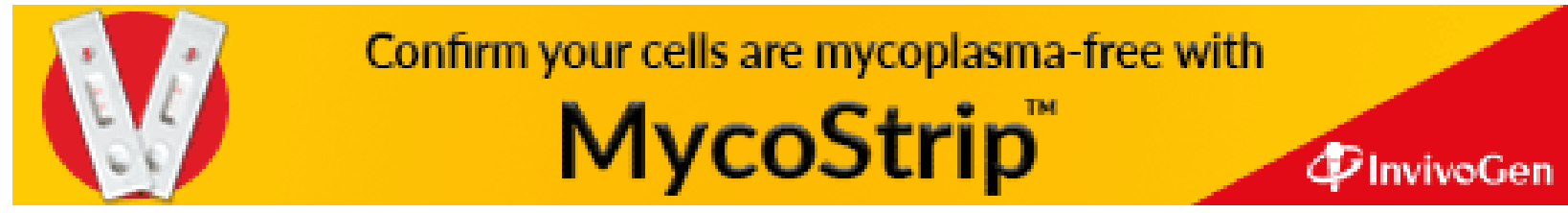

